\title{
A stochastic differential equation model for pest management
}

\author{
Xuewen Tan ${ }^{1,2^{*}}$, Sanyi Tang ${ }^{1}$, Xiaozhou Chen ${ }^{2}$, Lianglin Xiong ${ }^{2}$ and Xinzhi Liu ${ }^{3}$
}

${ }^{\text {*Correspondence: }}$ tanxw520@163.com

${ }^{1}$ College of Mathematics and Information Science, Shaanxi

Normal University, Xi'an, 710062, P.R. China

${ }^{2}$ School of Mathematics and Computer science, Yunnan Minzu University, Kunming, 650500, P.R. China

Full list of author information is available at the end of the article (c) The Author(s) 2017. This article is distributed under the terms of the Creative Commons Attribution 4.0 International License (http://creativecommons.org/licenses/by/4.0/), which permits unrestricted use, distribution, and reproduction in any medium, provided you give appropriate credit to the original author(s) and the source, provide a link to the Creative Commons license, and indicate if changes were made.

\section{Introduction}

Integrated pest management (IPM) was introduced and was widely practised recently [111]. IPM is a long time management strategy that uses a combination of cultural, biological and chemical tactics that reduce pests to tolerable levels with little cost to the grower and a minimal effect on the environment.

Especially, spraying of pesticides to crops is intended to reduce the population of pest, but can under some circumstances exacerbate a pest problem. This phenomenon, frequently called insecticide-induced resistance, has several possible mechanisms including reduction in herbivore-herbivore competition, physiological enhancement of pest fecundity, altered host plant nutrition, changes in pest behavior, and the killing of natural predators and parasites of the pest [12-18]. As a result, considerable emphasis has been placed on tactics other than chemical controls, including cultural, biological, and genetic methods and the deployment of crop varieties that are resistant to pests. Researchers have found that the cultural and biological pest controls are ecologically sound and could provide solutions that are sustainable in the long term $[19,20]$.

However, chemical pesticides may have long-term residual effects which reduce the populations of pest for several weeks, months or years. On the other hand, population dynamics in the real world is inevitably affected by environmental noises. So it is very important to study stochastic population systems to analyze the effect of environmental noises on population systems [21-27]. 
Based on the above discussions, this paper is to construct a stochastic integrated pest management (IPM) model with pesticides that have residual effects firstly. In the first place, we derive that the model has a global positive solution by using the mathematical induction method. On the other hand, the explicit expression of the pest-eradication solution is given. Moreover, in order to show residual effects, the sufficient condition for pest extinction and the global attractivity of the pest solution when the natural enemy disappears are established. Furthermore, we establish sufficient conditions for weak persistence in the mean and also show that the solution of stochastic IPM models is stochastically ultimately bounded under some conditions. The methods may have applications for a wider range of aspects of theoretical biology.

\section{The killing efficiency rate with pulses of chemical control}

It is universally acknowledged that the residual effects of pesticides exert desirable impacts on pest control. Therefore, a more realistic and appropriate method for modeling chemical control in such a case is to use continuous or piecewise-continuous periodic functions which affect the growth rate in the logistic model [1-3]. Such periodic functions make the growth rate fluctuate so that it decreases significantly, or even takes on negative values when the effects of pesticides have disappeared, but increases again during the recovery stage.

As mentioned above, more and more pesticides have a residual effect, which decays with time because of the degradation of pesticide and natural clearance $[4,5]$. In general, the killing efficiency rate $h(t)$ can take on various periodic (period $T$ ) forms including the step function of the form [5]

$$
h(t)= \begin{cases}m, & n T \leq t \leq n T+\tau, \\ 0, & n T+\tau \leq t \leq(n+1) T,\end{cases}
$$

where $\tau$ is the duration of pesticide residues. For simplicity, we will focus on the step function $h(t)$ of the form defined by (2.1) throughout this paper (see Figure 1).

\section{The stochastic IPM model with residual effects of pesticides}

The complex interactions amongst pests, natural enemies and pesticides can be enhanced with mathematical modeling [4, 5]. Modeling advances regarding IPM strategies include analyses of continuous and discrete predator-prey models [6-10]. In addition, discrete

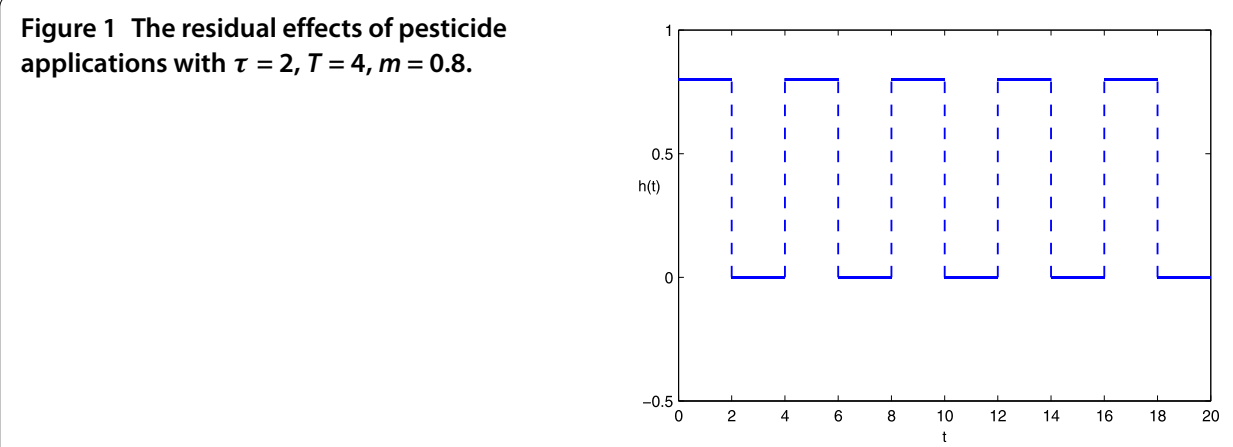


host-parasitoid models have been used to study four cases involving the timing of pesticide applications when these also led to the death of the parasitoids [16].

In [18], they first take into account the simplest case where in each impulsive period $T$ there is a pesticide application, so the killing efficiency rate function can be formulated by the exponentially decaying piecewise periodic function. Further, in each impulsive period $T$, there is an introduction constant $R$ for the natural enemies which does not depend on the size of the populations. These assumptions result in the following pest-natural enemy model:

$$
\left\{\begin{array}{l}
\frac{d P(t)}{d t}=P(t)(r-b P(t))-h(t) P(t)-\beta P(t) N(t), \\
\frac{d N(t)}{d t}=\lambda \beta P(t) N(t)-d N(t), \\
N\left(n T^{+}\right)=N(n T)+R, \quad t=n T,
\end{array}\right\} t \neq n T,
$$

where $P(t)$ and $N(t)$ are the population density of the pest and the natural enemy at time $t$, respectively, and the function $h(t)$ is defined by (2.1), $r$ represents the intrinsic growth rate and $r / b$ is the carrying capacity parameter, $\beta$ denotes the attack rate of the predator, $\lambda$ represents conversion efficiency and $d$ is the predator mortality rate. The same model (3.1) but without any residual effects of the pesticides on the pest (i.e., only an instantaneous killing efficiency was considered) has been investigated, see [4, 5] for details.

On the other hand, in the real world, the growth of species usually suffers some discrete changes of relatively short time interval at some fixed times with some natural and man-made factors, such as drought, harvesting, fire, earthquake, flooding, deforestation, hunting and so on. These phenomena cannot be considered with certainty, so in this case, the determined model (3.1) cannot be applicable. In order to describe these phenomena more accurately, some authors considered the stability of stochastic differential equation (SDE) [21]. However, as far as our knowledge is concerned, there is very little amount of work on the impulsive stochastic population model. By now, there have been no results related to the stochastic IPM model with residual effects of pesticides with impulsive effects.

Inspired by the above discussions, we develop the following stochastic IPM model with residual effects of pesticides with impulsive effects:

$$
\left\{\begin{array}{l}
d P(t)=[P(t)(r-b P(t))-h(t) P(t)-\beta P(t) N(t)] d t+\alpha_{1} P(t) d B_{1}(t), \\
d N(t)=\lambda \beta P(t) N(t) d t-d N(t) d t+\alpha_{2} N(t) d B_{2}(t), \\
N\left(n T^{+}\right)=N(n T)(1+q(n T)), \quad t=n T,
\end{array}\right\} t \neq n T,
$$

where $\alpha_{1}$ and $\alpha_{2}$ are the coefficients of the effects of environmental stochastic perturbations on the pest and the natural enemy, $B_{i}(t), i=1,2$, are the standard Brownian motions independent of each other and with the initial condition $B_{i}(0)=0, i=1,2, q(n T)$ represents the proportion of releasing natural enemies at time $n T$, the other parameters are the same as in model (3.1).

\subsection{Mathematical analysis of the pest-free solution}

One of the main purposes of investigating model (3.2) is to determine how to implement an IPM strategy so that the population goes extinct eventually. To do this, we first need to 
consider the following subsystem:

$$
\left\{\begin{array}{l}
d N(t)=-d N(t) d t+\alpha_{2} N(t) d B_{2}(t), \quad t \neq n T, \\
N\left(n T^{+}\right)=N(n T)(1+q(n T)), \quad t=n T, \\
N\left(0^{+}\right)=N(0) .
\end{array}\right.
$$

In order obtain the globally positive pest-free solution, we will give a solution of the definition of an impulsive stochastic differential equation.

Throughout this paper, let $\left(\Omega, \mathscr{F},\left\{\mathscr{F}_{t}\right\}_{t \geq 0}, \mathscr{P}\right)$ be a complete probability space with a filtration $\left\{\mathscr{F}_{t}\right\}_{t \geq 0}$ satisfying the usual conditions. Let $B(t)$ denote a standard Brownian motion defined on this probability space. Moreover, we always assume that a product equals unity if the number of factors is zero.

Definition 1 ([21]) Consider the following ISDE:

$$
\left\{\begin{array}{l}
d X(t)=f(t, X(t)) d t+\sigma(t, X(t)) d B(t), \quad t \neq n T \\
X\left(n T^{+}\right)=(1+q(n T)) X(n T), \quad t=n T
\end{array}\right.
$$

with the initial condition $X(0)$. A stochastic process $X(t)=\left(X_{1}(t), X_{2}(t), \ldots, X_{n}(t)\right)^{\tau}, t \in R_{+}$, is said to be s solution of ISDE (3.4) if:

(i) $X(t)$ is $\mathscr{F}_{t}$-adapted and continuous on $(0, T)$ and each interval $(n T,(n+1) T) \subset R_{+}$, $n \in \mathcal{N} ; f(t, X(t)) \in \mathscr{L}^{1}\left(R_{+} ; R_{n}\right), \sigma(t, X(t)) \in \mathscr{L}^{2}\left(R_{+} ; R_{n}\right)$, where $\mathscr{L}^{n}\left(R_{+} ; R_{n}\right)$ is all $R^{n}$-valued measurable $\mathscr{F}_{t}$-adapted processes $g(t)$ satisfying $\int_{0}^{\zeta}|g(t)|^{n} d t<\infty$ a.s. (almost surely) for every $\zeta>0$;

(ii) for each $n T, n \in \mathcal{N}, X\left(n T^{+}\right)=\lim _{t \rightarrow n T^{+}} X(t)$ and $X\left(n T^{-}\right)=\lim _{t \rightarrow n T^{-}} X(t)$ exist and $X(n T)=X\left(n T^{-}\right)$with probability one;

(iii) for almost all $t \in(0, T], P(t)$ obeys the integral equation

$$
X(t)=X(0)+\int_{0}^{t} f(s, X(s)) d s+\int_{0}^{t} \sigma(s, X(s)) d B(s) .
$$

And for almost all $t \in(n T,(n+1) T], n \in \mathcal{N}, X(t)$ obeys the integral equation

$$
X(t)=X\left(n T^{+}\right)+\int_{n T}^{t} f(s, X(s)) d s+\int_{n T}^{t} \sigma(s, X(s)) d B(s) .
$$

Moreover, $X(t)$ satisfies the impulsive conditions at each $t=n T, n \in \mathcal{N}$ with probability one.

Let us state and prove the following result.

Theorem 1 For any initial value $N\left(0^{+}\right)=N(0)$, there exists a unique positive solution $N(t)$ to ISDE (3.3) a.s., which is global and explicit

$$
N(t)=\prod_{0<n T<t}(1+q(n T)) N(0) \exp \left\{\left(-d-0.5 \alpha_{2}^{2}\right) t+\alpha_{2} B(t)\right\} .
$$

Proof Consider a Lyapunov function $V(t)$ defined by

$$
V(N(t), t)=\ln N(t), \quad t \geq 0
$$


Making use of Itô's formula

$$
d V(N(t), t)=\frac{d N(t)}{N(t)}-\frac{1}{2} \frac{(d N(t))^{2}}{N^{2}(t)}
$$

we can obtain that

$$
d(\ln N(t))=\left(-d-0.5 \alpha_{2}^{2}\right) d t+\alpha_{2} d B_{2}(t)
$$

Suppose $t \in(n T,(n+1) T], n \in \mathcal{N}$. Integrating both sides from $n T$ to $t$, one can see that

$$
\ln N(t)-\ln N(n T)=\left(-d-0.5 \alpha_{2}^{2}\right)(t-n T)+\alpha_{2}(B(t)-B(n T))
$$

Applying the impulse condition $N\left(n T^{+}\right)=(1+q(n T)) N(n T)$, we can obtain that

$$
N(t)=(1+q(n T)) N(n T) \exp \left\{\left(-d-0.5 \alpha_{2}^{2}\right)(t-n T)+\alpha_{2}(B(t)-B(n T))\right\} .
$$

Applying the mathematical induction method, we get

$$
N(t)=\prod_{0<n T<t}(1+q(n T)) N(0) \exp \left\{\left(-d-0.5 \alpha_{2}^{2}\right) t+\alpha_{2} B(t)\right\}
$$

\subsection{Mathematical analysis of chemical control only}

As the natural enemy disappeared, one of the main purposes of investigating model (3.2) is to determine under what circumstances the pest goes extinct eventually. To do this, we first need to consider the following subsystem:

$$
d P(t)=[P(t)(r-b P(t))-h(t) P(t)] d t+\alpha_{1} P(t) d B_{1}(t)
$$

In order to establish a sufficient condition for pest extinction and prove the global attractivity of solution of model (3.5), we introduce some useful definitions and lemmas.

Definition 2 If $N_{1}(t), N_{2}(t)$ are two arbitrary solutions of model (3.3) with the initial values $N_{1}(0), N_{2}(0)>0$, respectively. If $\lim _{t \rightarrow \infty}\left|N_{1}(t)-N_{2}(t)\right|=0$ a.s., then Eq. (3.3) is globally attractive.

Lemma 1 If $N(t)$ is a solution of (3.3) without impulsive effects for any initial value $N(0)=N_{0}>0$. If Assumption 1 holds, then almost every sample path of $N(t)$ is uniformly continuous for $t \geq 0$.

Lemma 2 Iff is a non-negative function defined on $R_{+}$such that $f$ is integrable on $R_{+}$and is uniformly continuous on $R_{+}$, then $\lim _{t \rightarrow \infty} f(t)=0$.

In the following, we will give our main result.

Theorem 2 If $r-0.5 \alpha_{1}^{2}-\frac{m \tau}{T}<0$, then the solution $P(t)$ of SDE (3.5) with any positive initial value has $\lim _{t \rightarrow \infty} P(t)=0$. 
Proof Making use of Itô's formula, we derive the following form in model (3.5):

$$
d(\log P(t))=\left[r-0.5 \alpha_{1}^{2}-h(t)-b P(t)\right] d t+\alpha_{1} d B(t)
$$

Integrating both sides from 0 to $t$, we have

$$
\begin{aligned}
\log P(t) & =\log P_{0}+\int_{0}^{t}\left[r-0.5 \alpha_{1}^{2}-h(t)\right] d s-b \int_{0}^{t} P(s) d s+\alpha_{1} \int_{0}^{t} d B(s) \\
& \leq \log P_{0}+\int_{0}^{t}\left[r-0.5 \alpha_{1}^{2}-h(t)\right] d s+M_{1}(t)
\end{aligned}
$$

where $M_{1}(t)$ is a martingale defined by

$$
M_{1}(t)=\alpha_{1} \int_{0}^{t} d B(s)
$$

The quadratic variation of this martingale is

$$
\left\langle M_{1}(t), M_{1}(t)\right\rangle=\alpha_{1}^{2} t
$$

Making use of the strong law of large numbers for local martingales (see [23], p.26) leads to

$$
\lim _{t \rightarrow \infty} M_{1}(t) / t=0
$$

According to the hypothesis,

$$
\lim _{t \rightarrow \infty} \sup \frac{\log P(t)}{t} \leq \lim _{t \rightarrow \infty} \sup \frac{\int_{0}^{t}\left[r-0.5 \alpha_{1}^{2}-\frac{m \tau}{T}\right] d s}{t} \doteq \lambda^{*}<0
$$

hence, we can derive that

$$
\lim _{t \rightarrow \infty} P(t)=0
$$

Theorem 3 The solution of model (3.5) is globally attractive.

Proof Let $P_{1}(t), P_{2}(t)$ be two arbitrary solutions of model (3.5) with the initial values $P_{1}(0), P_{2}(0)>0$, respectively. Suppose that the solution of the equation

$$
d P(t)=[P(t)(r-b P(t))-h(t) P(t)] d t+\alpha_{1} P(t) d B_{1}(t) \quad \text { with } P(0)=P_{1}(0)
$$

is $P_{1}(t)$ and the solution of the equation

$$
d P(t)=[P(t)(r-b P(t))-h(t) P(t)] d t+\alpha_{1} P(t) d B_{1}(t) \quad \text { with } P(0)=P_{2}(0)
$$

is $P_{2}(t)$. Consider a Lyapunov function $V(t)$ defined by

$$
V(t)=\left|\ln P_{1}(t)-\ln P_{2}(t)\right|, \quad t \geq 0 .
$$

Then $V(t)$ is continuous. So a direct calculation of the right differential $d^{+} V(t)$ of $V(t)$ 
along the solutions leads to

$$
\begin{aligned}
d^{+} V(t) & =\operatorname{sgn}\left(P_{1}(t)-P_{2}(t)\right) d\left(\ln P_{1}(t)-\ln P_{2}(t)\right) \\
& =\operatorname{sgn}\left(P_{1}(t)-P_{2}(t)\right)\left[-b\left(P_{1}(t)-P_{2}(t)\right)\right] d t \\
& =-b\left|P_{1}(t)-P_{2}(t)\right| d t .
\end{aligned}
$$

Integrating both sides and then taking the expectation, we have that

$$
V(t)-V(0)=-b \int_{0}^{t}\left|P_{1}(s)-P_{2}(s)\right| d s .
$$

Consequently, it gives that

$$
V(t)+b \int_{0}^{t}\left|P_{1}(s)-P_{2}(s)\right| d s=V(0)<\infty .
$$

Then it follows from $V(t) \geq 0$ that $\left|P_{1}(s)-P_{2}(s)\right| \in L^{1}[0, \infty)$. Combining Lemma 1 with Lemma 2 can derive that

$$
\lim _{t \rightarrow \infty}\left|P_{1}(t)-P_{2}(t)\right|=0
$$

\subsection{The persistence and stochastically ultimate boundedness}

In order to investigate the persistence and stochastically ultimate boundedness, we need to guarantee the existence and uniqueness of the positive solution. In the following, we illustrate the definitions of solutions of system (3.2).

Theorem 4 Model (3.2) has a unique solution $(P(t), N(t))$ on $t>0$ for any given initial value $(P(0), N(0)) \in R_{+}^{2}=\left\{(P(t), N(t)) \in R_{+}^{2} \mid P(t)>0, N(t)>0\right\}$ and the solution will remain in $R_{+}^{2}$ a.s.

Proof According to [21], consider the following SDE without impulse effects:

$$
\left\{\begin{aligned}
d P_{1}(t)= & {\left[P_{1}(t)\left(r-b P_{1}(t)\right)-h(t) P_{1}(t)-\beta \prod_{0<n T<t}(1+q(n T)) P_{1}(t) N_{1}(t)\right] d t } \\
& +\alpha_{1} P_{1}(t) d B_{1}(t), \\
d N_{1}(t)= & \lambda \beta P_{1}(t) N_{1}(t) d t-d N_{1}(t) d t+\alpha_{2} N_{1}(t) d B_{2}(t)
\end{aligned}\right.
$$

with the initial value $\left(P_{1}(0), N_{1}(0)\right)=(P(0), N(0))$. Model (3.6) has a unique global positive solution $\left(P_{1}(t), N_{1}(t)\right)$. Let $N(t)=\prod_{0<n T<t}(1+q(n T)) N_{1}(t)$, we may prove that $(P(t), N(t))$ is the solution to model (3.2) with the initial value $(P(0), N(0))$. Because of the first equation of (3.2), we only consider the second equation of model (3.2), by the fact that $N(t)$ is continuous on $(0, T]$ and each interval $t \in(n T,(n+1) T] \subset[0, \infty), n \in \mathcal{N}$ and for $t \neq n T$

$$
\begin{aligned}
d N(t) & =d\left[\prod_{0<n T<t}(1+q(n T)) N_{1}(t)\right]=\prod_{0<n T<t}(1+q(n T)) d N_{1}(t) \\
& =\lambda \beta \prod_{0<n T<t}(1+q(n T)) P_{1}(t) N_{1}(t) d t-d \prod_{0<n T<t}(1+q(n T)) N_{1}(t) d t
\end{aligned}
$$




$$
\begin{gathered}
\quad+\alpha_{2} \prod_{0<n T<t}(1+q(n T)) N_{1}(t) d B_{2}(t) \\
=\lambda \beta P(t) N(t) d t-d N(t) d t+\alpha_{2} N(t) d B_{2}(t) .
\end{gathered}
$$

Moreover, for every $n \in \mathcal{N}$ and $t \neq n T$,

$$
\begin{aligned}
N\left(n T^{+}\right) & =\lim _{t \rightarrow n T^{+}} N(t) \\
& =\lim _{t \rightarrow n T^{+}} \prod_{0<i T<t}(1+q(i T)) N_{1}(t) \\
& =\lim _{t \rightarrow n T^{+}} \prod_{0<i T \leq n T}(1+q(i T)) N_{1}\left(n T^{+}\right) \\
& =(1+q(n T)) \prod_{0<i T<n T}(1+q(i T)) N_{1}(n T) \\
& =(1+q(n T)) N(n T) .
\end{aligned}
$$

On the other hand,

$$
\begin{aligned}
N\left(n T^{-}\right) & =\lim _{t \rightarrow n T^{-}} N(t) \\
& =\lim _{t \rightarrow n T^{-}} \prod_{0<i T<t}(1+q(i T)) N_{1}(t) \\
& =\lim _{t \rightarrow n T^{+}} \prod_{0<i T<n T}(1+q(i T)) N_{1}\left(n T^{-}\right) \\
& =\prod_{0<i T<n T}(1+q(i T)) N_{1}(n T)=(1+q(n T)) N(n T) .
\end{aligned}
$$

Theorem 4 indicates that model (3.2) has a unique global positive solution. This main result allows us to further examine how the solution varies in $R_{+}^{2}$ in more detail. Now let us further examine how this solution pathwisely moves in $R_{+}$.

Assumption 1 There are two positive constants $L$ and $U$ such that $L \leq \prod_{0<n T<t}(1+$ $q(n T)) \leq U$.

Theorem 5 Under Assumption 1, the solution $(P(t), N(t))$ of model (3.2) satisfies the following inequalities:

$$
\lim _{t \rightarrow \infty} \sup E\left[\sqrt{\left(P_{1}^{2}(s)+N_{1}^{2}(s)\right)}\right] \leq \mathcal{K} \quad\left(\mathcal{K} \in R^{+}\right)
$$

Proof According to model (3.6), we define

$$
A=\left[\begin{array}{cc}
-b & -\beta \prod_{0<n T<t}(1+q(n T)) \\
\lambda \beta & 0
\end{array}\right],
$$

then there exist two constants $v_{1}$ and $v_{2}$ (see) such that

$$
\lambda_{\max }\left(\widetilde{C} A+A^{T} \widetilde{C}\right)<0,
$$


where $\widetilde{C}=\operatorname{diag}\left(c_{1}, c_{2}\right)$, and $\lambda_{\max }\left(\widetilde{C} A+A^{T} \widetilde{C}\right)$ is the largest eigenvalue of $\widetilde{C} A+A^{T} \widetilde{C}$. In order to show this statement, define a $C^{2}$-function V: $R_{+}^{2} \rightarrow R_{+}$by

$$
V\left(P_{1}(t), N_{1}(t)\right)=c_{1} P_{1}(t)+c_{2} N_{1}(t)
$$

In view of Itồs formula, we get

$$
d V\left(P_{1}(t), N_{1}(t)\right)=L V\left(P_{1}(t), N_{1}(t)\right) d t+c_{1} \alpha_{1} P_{1}(t) d B_{1}(t) c_{2} \alpha_{2} N_{1}(t) d B_{2}(t),
$$

where $L V\left(P_{1}(t), N_{1}(t)\right): R_{+}^{2} \rightarrow R_{+}$is defined by

$$
\begin{aligned}
L V & \left(P_{1}(t), N_{1}(t)\right) \\
= & c_{1} P_{1}(t)\left[r-b P_{1}(t)-h(t)-\beta \prod_{0<n T<t}(1+q(n T)) N_{1}(t)\right] \\
& +c_{2} N_{1}(t)\left(\lambda \beta P_{1}(t)-d\right) \\
= & r c_{1} P_{1}(t)-d c_{2} N_{1}(t)+\frac{1}{2}\left(P_{1}(t), N_{1}(t)\right)\left(\widetilde{C} A+A^{T} \widetilde{C}\right)\left(P_{1}(t), N_{1}(t)\right)^{T} \\
\leq & r c_{1} P_{1}(t)-d c_{2} N_{1}(t)+\frac{1}{2} \lambda_{\max }\left(\widetilde{C} A+A^{T} \widetilde{C}\right)\left(P_{1}^{2}(t)+N_{1}^{2}(t)\right) .
\end{aligned}
$$

In the following, consider a Lyapunov function $V_{1}(t)$ defined by

$$
V_{1}(t)=e^{t} V\left(P_{1}(t), N_{1}(t)\right), \quad t \geq 0 .
$$

Making use of Itô’s formula, we get

$$
\begin{aligned}
d\left[e^{t} V\left(P_{1}(t), N_{1}(t)\right)\right]= & e^{t} V\left(P_{1}(t), N_{1}(t)\right) d t+e^{t} V\left(P_{1}(t), N_{1}(t)\right) \\
= & \left(c_{1} P_{1}(t)-c_{2} N_{1}(t)\right) e^{t} d t+e^{t}\left(L V\left(P_{1}(t), N_{1}(t)\right)\right) d t \\
& +c_{1} \alpha_{1} P_{1}(t) d B_{1}(t)-c_{2} \alpha_{2} N_{1}(t) d B_{2}(t) \\
\leq & e^{t}\left[c_{1} P_{1}(t)-c_{2} N_{1}(t)+r c_{1} P_{1}(t)-d c_{2} N_{1}(t)\right. \\
& \left.+\frac{1}{2} \lambda_{\max }\left(\widetilde{C} A+A^{T} \widetilde{C}\right)\left(P_{1}^{2}(t)+N_{1}^{2}(t)\right)\right] d t \\
& +e^{t}\left[c_{1} \alpha_{1} P_{1}(t) d B_{1}(t)-c_{2} \alpha_{2} N_{1}(t) d B_{2}(t)\right] .
\end{aligned}
$$

Let

$$
\begin{aligned}
g\left(P_{1}(t), N_{1}(t)\right) \doteq & c_{1} P_{1}(t)-c_{2} N_{1}(t)+r c_{1} P_{1}(t)-d c_{2} N_{1}(t) \\
& +\frac{1}{2} \lambda_{\max }\left(\widetilde{C} A+A^{T} \widetilde{C}\right)\left(P_{1}^{2}(t)+N_{1}^{2}(t)\right) .
\end{aligned}
$$

It is easy to see that there exists a constant $\mathcal{K}_{1}>0$ such that $g\left(P_{1}(t), N_{1}(t)\right) \leq \mathcal{K}_{1}$, so we have that

$$
d\left[e^{t} V\left(P_{1}(t), N_{1}(t)\right)\right] \leq \mathcal{K}_{1} e^{t} d t+e^{t}\left[c_{1} \alpha_{1} P_{1}(t) d B_{1}(t)-c_{2} \alpha_{2} N_{1}(t) d B_{2}(t)\right] .
$$


Integrating from 0 to $t$ and then taking expectations, one can get

$$
e^{t} E\left[V\left(P_{1}(t), N_{1}(t)\right)\right] \leq V\left(P_{1}(0), N_{1}(0)\right)+\mathcal{K}_{1}\left(e^{t}-1\right) .
$$

Taking superior limit of both sides, we get

$$
\lim _{t \rightarrow \infty} \sup E\left[V\left(P_{1}(t), N_{1}(t)\right)\right] \leq \mathcal{K}_{1} .
$$

On the other hand,

$$
\sqrt{\left(P_{1}^{2}(t)+N_{1}^{2}(t)\right)} \leq P_{1}(t)+N_{1}(t) \leq V\left(P_{1}(t), N_{1}(t)\right) / \min \left\{c_{1}, c_{2}\right\}
$$

Consequently,

$$
\begin{aligned}
\lim _{t \rightarrow \infty} \sup E\left[\sqrt{\left(P_{1}^{2}(t)+N_{1}^{2}(t)\right)}\right] & \leq \lim _{t \rightarrow \infty} \sup E\left[V\left(P_{1}(t), N_{1}(t)\right)\right] / \min \left\{c_{1}, c_{2}\right\} \\
& \leq \mathcal{K}_{1} / \min \left\{c_{1}, c_{2}\right\} \doteq \mathcal{K} .
\end{aligned}
$$

For better discussion later, we give several definitions, then try to explore sufficient conditions for them.

Definition 3 If $\lim _{t \rightarrow \infty} \sup \frac{\int_{0}^{t} P(s) d s}{t}>0$ a.s., then the species $P(t)$ is weakly persistent in the mean.

Definition 4 A solution $\mathscr{Z}(t)=(P(t), N(t))$ of Eq. (3.2) is said to be stochastically ultimately bounded if $\forall \epsilon \in(0,1), \exists \delta=\delta^{*}>0$ such that

$$
\lim _{t \rightarrow \infty} \sup \mathscr{P}[|\mathscr{Z}(t)|>\delta]<\epsilon
$$

for any initial value $(P(0), N(0)) \in R_{+}^{2}$.

We are now in a position to prove persistence. For the pest $P(t)$ and the natural enemy $N(t)$, we have the following results.

\section{Theorem 6}

(i) If $r-0.5 \alpha_{1}^{2}-\frac{m \tau}{T}>0, \lim _{t \rightarrow \infty} \sup \frac{\sum_{0<n T<t} \ln (1+q(n T))}{t}<-d+0.5 \alpha_{2}^{2}$, then the pest $P(t)$ is weakly persistent in the mean.

(ii) If $\lambda \beta\left(r-0.5 \alpha_{1}^{2}-\frac{m \tau}{T}\right)+b \lim _{t \rightarrow \infty} \sup \frac{\sum_{0<n T<t} \ln (1+q(n T))}{t}+d-0.5 \alpha_{2}^{2}>0$, and $\lim _{t \rightarrow \infty} \sup \frac{\sum_{0<n T<t} \ln (1+q(n T))}{\ln t}<\infty$, then the natural enemy $N(t)$ will be weakly persistent in the mean.

Proof For the pest $P(t)$, consider a Lyapunov function $V(t)$ defined by

$$
V(t)=\ln P(t), \quad t \geq 0
$$


Applying Itô’s formula to the first equation of Eq. (3.2) yields

$$
d \ln P(t)=\frac{d P(t)}{P(t)}-\frac{(d P(t))^{2}}{2 P^{2}(t)}=r-h(t)-0.5 \alpha_{1}^{2}-b P(t)-\beta N(t)+\alpha_{1} d B_{1}(t) .
$$

Integrating both sides from 0 to $t$ and both dividing by $t$, one can see that

$$
\frac{\ln [P(t) / P(0)]}{t}=r-0.5 \alpha_{1}^{2}-\frac{m \tau}{T}-\frac{b \int_{0}^{t} P(s) d s}{t}-\frac{\beta \int_{0}^{t} N(s) d s}{t}+\frac{M_{1}(t)}{t} .
$$

In the same way,

$$
\frac{\ln [N(t) / N(0)]}{t}=\frac{\sum_{0<n T<t} \ln (1+q(n T))}{t}+d-0.5 \alpha_{2}^{2}+\frac{\lambda \beta \int_{0}^{t} P(s) d s}{t}+\frac{M_{2}(t)}{t},
$$

where $M_{i}(t)=\int_{0}^{t} \alpha_{i} d B_{i}(s), i=1,2$. Then $M_{i}(t)$ is a local martingale whose quadratic variation $\left\langle M_{i}(t), M_{i}(t)\right\rangle=\int_{0}^{t} \alpha_{i}^{2} d(s)$. Making use of the strong law of large numbers for local martingales ([23], p.26) leads to

$$
\lim _{t \rightarrow \infty} \frac{M_{i}(t)}{t}=0, \quad \text { a.s. } i=1,2 \text {. }
$$

Case (i). By Theorem 5 , we have that $\lim _{t \rightarrow \infty} \sup \frac{P(t)}{t}<0$ a.s. Then taking the superior limit for (3.7) leads to

$$
\lim _{t \rightarrow \infty} \sup \left(\frac{b \int_{0}^{t} P(s) d s}{t}-\frac{\beta \int_{0}^{t} N(s) d s}{t}\right)>r-0.5 \alpha_{1}^{2}-\frac{m \tau}{T} \quad \text { a.s. }
$$

There $\lim _{t \rightarrow \infty} \sup \frac{\int_{0}^{t} P(s) d s}{t}>0$ a.s. In fact, for $\forall \omega \in\left\{\lim _{t \rightarrow \infty} \sup \frac{P(t, \omega)}{t}=0\right\}$, it follows from (3.10) that $\lim _{t \rightarrow \infty} \sup \frac{N(t, \omega)}{t}>0$. On the other hand, taking the superior limit for (3.8) and using $\lim _{t \rightarrow \infty} \sup \frac{P(t, \omega)}{t}=0$ a.s., according to the hypothesis (i), lead to

$$
\lim _{t \rightarrow \infty} \sup \frac{\ln N(t, \omega)}{t} \leq \lim _{t \rightarrow \infty} \sup \frac{\sum_{0<n T<t} \ln (1+q(n T))}{t}+d-0.5 \alpha_{2}^{2}<0 .
$$

This is to say $\lim _{t \rightarrow \infty} N(t, \omega)=0$, which is a contraction. So we have $\lim _{t \rightarrow \infty} \sup \frac{P(t, \omega)}{t}>0$ a.s.

Case (ii). Multiplying (3.7) and (3.8) by $\lambda \beta$ and $b$, respectively, we find

$$
\begin{aligned}
\lambda \beta & \frac{\ln [P(t) / P(0)]}{t}+b \frac{\ln [N(t) / N(0)]}{t} \\
= & \lambda \beta\left(r-0.5 \alpha_{1}^{2}-\frac{m \tau}{T}\right)+b\left(\frac{\sum_{0<n T<t} \ln (1+q(n T))}{t}+d-0.5 \alpha_{2}^{2}\right) \\
& -\frac{\lambda \beta^{2} \int_{0}^{t} N(s) d s}{t}+\frac{\lambda \beta M_{1}(t)}{t}+\frac{b M_{2}(t)}{t} .
\end{aligned}
$$

Using the strong law of large numbers for local martingales leads to

$$
\lim _{t \rightarrow \infty} \frac{\lambda \beta M_{1}(t)}{t}=0, \quad \lim _{t \rightarrow \infty} \frac{b M_{2}(t)}{t}=0 .
$$


Noting that $\lim _{t \rightarrow \infty} \frac{\ln P(t)}{t} \leq 0, \lim _{t \rightarrow \infty} \frac{\ln N(t)}{t} \leq 0$ a.s. and taking the superior limit for (3.11) lead to

$$
\begin{aligned}
& \lim _{t \rightarrow \infty} \sup \frac{\lambda \beta^{2} \int_{0}^{t} N(s) d s}{t} \\
& \quad \geq \lambda \beta\left(r-0.5 \alpha_{1}^{2}-\frac{m \tau}{T}\right)+b \lim _{t \rightarrow \infty} \sup \frac{\sum_{0<n T<t} \ln (1+q(n T))}{t}+d-0.5 \alpha_{2}^{2}>0 .
\end{aligned}
$$

Theorem 7 The solution $(P(t), N(t))$ of the equation system (3.2) is stochastically bounded.

Proof According to Theorem 5, we obtain

$$
\begin{aligned}
& \lim _{t \rightarrow \infty} \sup E \sqrt{P^{2}(t)+N^{2}(t)} \\
& \quad=\lim _{t \rightarrow \infty} \sup E \sqrt{P^{2}(t)+\left[\sum_{0<n T<t} \ln (1+q(n T)) N_{1}(t)\right]^{2}} \\
& \quad \leq \sqrt{\left(1+M^{2}\right)} \lim _{t \rightarrow \infty} \sup E \sqrt{P_{1}^{2}(t)+N_{1}^{2}(t)} \\
& \quad=\sqrt{\left(1+M^{2}\right)} \mathcal{K} \doteq U^{*} .
\end{aligned}
$$

Let $\delta=U^{*} / \epsilon$, by Chebyshev's inequality, we have

$$
\mathscr{P}\{|P(t)|>\delta\} \leq \frac{E \sqrt{P^{2}(t)+N^{2}(t)}}{\delta} .
$$

Taking the superior limit for both sides, we get

$$
\lim _{t \rightarrow \infty} \sup \mathscr{P}[|P(t)|>\delta]<\epsilon
$$

\section{Discussion}

In this paper, a simple stochastic mathematical model of IPM systems with pesticides that have residual effects is proposed and studied. Theorem 4 shows that our model has a global positive solution for any given positive initial value, and we obtain its explicit expression under the pest-eradication condition. Firstly, we consider chemical control only, with the other parameters left the same as in model (3.2). Secondly, under $r-0.5 \alpha_{1}^{2}-\frac{m \tau}{T}<0$, which implies that the pest will die out eventually, we also prove the global attractivity of the pest solution when the natural enemy disappears.

It follows from Theorem 6 that we prove the persistence of pest and natural enemy when they satisfy the conditions respectively. Fortunately, Theorem 7 proves that the solution of model (3.2) is stochastically bounded with $L \leq \prod_{0<n T<t}(1+q(n T)) \leq U(L$ and $U$ are positive constants).

Integrated pest management is a complex process. In fact, farmers and other pest managers usually control pests so that they cannot exceed the economic injury level. How to model an IPM strategy with residual effects of pesticides, taking account of the economic injury level, will also be studied in future research to include analyses of dynamical behavior of the models and the biological implications of the results. 


\section{Acknowledgements}

This work was supported by the National Natural Science Foundation of China (No.11601268, 11461082, 11461083, 31460297), the Educational Commission of Yunnan Province (2015Y223), and the Educational Commission of Hubei Province (Q20161212). Moreover, this work is partly supported by Key Laboratory of IOT Application Technology of Universities in Yunnan Province.

\section{Competing interests}

The authors declare that they have no competing interests.

\section{Authors' contributions}

All authors contributed equally and significantly in this paper. All authors read and approved the final manuscript.

\section{Author details}

${ }^{1}$ College of Mathematics and Information Science, Shaanxi Normal University, Xi'an, 710062, P.R. China. ${ }^{2}$ School of Mathematics and Computer science, Yunnan Minzu University, Kunming, 650500, P.R. China. ${ }^{3}$ Department of Applied Mathematics, University of Waterloo, Waterloo, Ontario N2L 3G1, Canada.

\section{Publisher's Note}

Springer Nature remains neutral with regard to jurisdictional claims in published maps and institutional affiliations.

Received: 8 December 2016 Accepted: 20 June 2017 Published online: 11 July 2017

\section{References}

1. Tang, S, Cheke, R: Stage-dependent impulsive models of integrated pest management (IPM) strategies and their dynamic consequences. J. Math. Biol. 50, 257-292 (2005)

2. Tang, S, Xiao, Y, Chen, L: Integrated pest management models and their dynamical behaviour. Bull. Math. Biol. 67, $115-135(2005)$

3. Tang, S, Xiao, Y, Cheke, R: Multiple attractors of host-parasitoid models with integrated pest management strategies: eradication, persistence and outbreak. Theor. Popul. Biol. 73, 181-197 (2008)

4. Liang, J, Tang, S: Optimal dosage and economic threshold of multiple pesticide applications for pest control. Math. Comput. Model. 51, 487-503 (2010)

5. Liang, J, Tang, S: Analytical methods for detecting pesticide switches with evolution of pesticide resistance. Math. Biosci. 245, 249-257 (2013)

6. Zhang, T, Zhang, J, Meng, X: Geometric analysis of a pest management model with Hollings type III functional response and nonlinear state feedback control. Nonlinear Dyn. 84, 1529-1539 (2016)

7. Zhao, W, Li, J, Zhang, T: Persistence and ergodicity of plant disease model with Markov conversion and impulsive toxicant input. Commun. Nonlinear Sci. Numer. Simul. 48, 70-84 (2017)

8. Zhang, T, Meng, X, Liu, R: Periodic solution of a pest management Gompertz model with impulsive state feedback control. Nonlinear Dyn. 78, 921-938 (2014)

9. Sun, K, Zhang, T, Tian, Y: Theoretical study and control optimization of an integrated pest management predator prey model with power growth rate. Math. Biosci. 279, 13-26 (2016)

10. Sun, $K$, Zhang, $T$, Tian, Y: Dynamics analysis and control optimization of a pest management predator-prey model with an integrated control strategy. Appl. Math. Comput. 292, 253-271 (2017)

11. Qin, W, Tang, S, Cheke, RA: The effects of resource limitation on a predator-prey model with control measures as nonlinear pulses. Math. Probl. Eng. 14, Article ID 450935 (2014)

12. Barclay, $\mathrm{H}$ : Models for pest control using predator release, habitat management and pesticide release in combination. J. Appl. Ecol. 19, 337-348 (1982)

13. Blower, S, Dowlatabadi, H: Sensitivity and uncertainty analysis of complex-models of disease transmission? An HIV model, as an example. Int. Stat. Rev. 62, 229-243 (1994)

14. Barlow, N, Moller, H, Beggs, J: A model for the effect of Sphecophaga vesparum as a biological control agent of the common wasp in New Zealand. J. Appl. Ecol. 33, 31-34 (1996)

15. Caltagirone, L, Doutt, R: The history of the vedalia beetle importation to California and its impact on the development of biological control. Annu. Rev. Entomol. 34, 1-16 (1989)

16. Inoue, M, Kamifukumoto, H: Scenarios leading to chaos in forced Lotka-Volterra model. Prog. Theor. Phys. 17, 930-937 (1984)

17. Kot, M, Sayler, G, Schultz, T: Complex dynamics in a model microbial system. Bull. Math. Biol. 54, 619-648 (1992)

18. Sabin, G, Summers, D: Chaos in a periodically forced predator-prey ecosystem model. Math. Biosci. 113, 91-113 (1993)

19. Upadhyay, R, Rai, V, lyengar, S: How do ecosystems respond to external perturbations? Chaos Solitons Fractals 11, 1963-1982 (2000)

20. Grasman, J, Van Herwaarden, O: A two-component model of host-parasitoid interactions: determination of the size of inundative releases of parasitoids in biological pest control. Math. Biosci. 169, 207-216 (2001)

21. Liu, M, Wang, K: On a stochastic logistic equation with impulsive perturbations. Comput. Math. Appl. 63, 871-886 (2012)

22. Li, X, Mao, X: Sufficient and necessary conditions of stochastic permanence and extinction for stochastic logistic populations under regime switching. J. Math. Anal. Appl. 376, 11-28 (2011)

23. Mao, X, Yuan, C: Stochastic Differential Equations with Markovian Switching. Imperial College Press, London (2006)

24. Haskell, C, Sacker, R: The stochastic Beverton-Holt equation and the M. Neubert conjecture. J. Dyn. Differ. Equ. 17, 4825-4844 (2005)

25. Hu, S: Stochastic Differential Equations. Science Press, Beijing (2007)

26. Wu, R, Wang, K: Asymptotic properties of a stochastic Lotka-Volterra cooperative system with impulsive perturbations. Nonlinear Dyn. 77, 807-817 (2014)

27. Wu, R, Wang, K: Asymptotic behavior of a stochastic non-autonomous predator-prey model with impulsive perturbations. Commun. Nonlinear Sci. Numer. Simul. 20, 965-974 (2015) 